\title{
Otomycosis at Fann Hospital in Dakar (Senegal): Prevalence and Mycological Study
}

\author{
Dieng $\mathrm{T}^{1 *}$, Sow $\mathrm{D}^{2}$, Tine $\mathrm{RC}^{1}$, Yatassaye $\mathrm{F}^{1}$, Dia $\mathbf{M}^{1}$, Sylla $\mathrm{K}^{1}$, Lelo $\mathrm{S}^{1}$ and \\ Dieng $\mathbf{Y}^{1}$ \\ ${ }^{1}$ Department of Parasitology-Mycology, University Cheikh Anta DIOP, Senegal \\ ${ }^{2}$ Department of Parasitology-Mycology, University Gaston Berger, Senegal
}

*Corresponding author: Thérèse Dieng, Department of Parasitology-Mycology, Faculty of Medicine, Pharmacy and Odontology, University Cheikh Anta DIOP, Avenue Cheikh Anta Diop, Dakar, Senegal, Tel : +22177 65420 49; Email : therese.dieng@ucad.edu.sn

\author{
Research Article \\ Volume 3 Issue 2 \\ Received Date: August 03, 2020 \\ Published Date: September 22, 2020 \\ DOI: $10.23880 /$ oajmms-16000129
}

\section{Abstract}

Otomycosis are auricular infections caused by microscopic fungi which develop essentially in the external ear canal. Their incidence has increased these past years with the emergence of predisposing factors such as the repetitive use of broad -spectrum antibiotics. The objectives of this study were to determine the prevalence of otomycosis at Fann National University Hospital in Dakar and to identify the fungal species responsible for that. This descriptive retrospective study held from October 2011 to December 2017 at 136 patients with clinically suspected otomycosis. External auditory canal exudates collected with swabs in each patient were observed by direct microscopic examination and cultured in Sabouraud Chloramphenicol medium with and without Actidione at $30-37^{\circ} \mathrm{C}$. The strains were identified on the basis of macroscopic, microscopic and physiological characteristics. The mycological examination was positive in 59 patients, that is to say, a prevalence of $43.4 \%$. The positivity rate was significantly higher among women $(52.6 \%)$ than among men $(31 \%)(\mathrm{p}=0.012)$. This rate was significantly higher among patients suffering from otalgia $(75.7 \%)$ than among others $(\mathrm{p}=0.023)$. On the mycological plan, Aspergillus fumigatus and Candida albicans were the species the most isolated (26.3\%) followed by Aspergillus niger (22.8\%). These results show that fungal agents have an important place in the etiologies of the external otitis at Fann Hospital CHNU. Hence the interest of the mycological diagnosis for a better treatment of patients.

Keywords: Otomycosis; Prevalence; Aspergillus; Candida; Senegal

\section{Introduction}

Otomycosis are auricular infections caused by microscopic fungi which develop essentially in the external ear canal but which can also invade the inside of the ear. According to some studies, the otomycosis would represent 5 to $10 \%$ of the overall internal earaches. The factors which are more likely to cause otomycosis are mainly heat, moisture, poor body hygiene, bathing or diving in cool water or sea water, and using antibiotics for an earache caused by bacteria [1-4]. In these otomycosis, the fungi can cause either a real initial invasion or a secondary invasion to some tissue defects stemming from primary bacterial infection [5-6].
The frequency and the dosage of the implied agents vary according to the different geographical areas. However, the species belonging to the genus Candida and Aspergillus are generally the agents the most encountered [7]. The incidence of these infections has increased these past years due to the use of antibiotics at a large scale to cure bacterial earache, as well as other factors related to the shifts of immunity [7]. The diagnosis of fungal earache is based on the clinical and mycological examinations. Symptomatology on the basis of pruritus, otorrhea and earache, being a little specific, the mycological diagnosis identifying the responsible agent enables to confirm the diagnosis and thus putting in place an accurate cure. In Senegal, there are few data about the 


\section{Open Access Journal of Mycology \& Mycological Sciences}

epidemiological and etiological agents of otomycosis. The purpose of that study was to determine the prevalence of otomycosis diagnosed at the laboratory of Parasitology Mycology of Fann National University Hospital and to identify the responsible fungal agents.

\section{Materials and Methods}

We have conducted a descriptive retrospective survey going from October 2011 to December 2017. This survey implied 136 patients checked at the ORL clinic at Fann Hospital and for whom an auricular sample was subject to a mycological examination done at the Parasitology- Mycology laboratory.

\section{Sampling}

Samples were taken without antibiotics therapy and any antifungal treatment. The external ear canal was first cleaned with a wet swab and the auricular sample has been taken through swabbing using another sterile swab filled at $0.9 \%$ with sterile physiological water or dry in case of otorrhea. The samples were taken at once to the lab in order to do the mycological examination.

\section{Mycological Examination}

At first we took the swab out of the physiological water contained in the sterile test tube. We then started the microscopic screening of a drop of that physiological water between blade and lamella with $\mathrm{x} 10$ and $\mathrm{x} 40$ zooms and a Sabouraud chloramphenicol (SC) and Sabouraud Chloramphenicol-Actidione (SCA) media. These media were inoculated by flooding thanks to a Pasteur pipette sterilized from physiological water in which the sample was taken out. The test tubes were incubated at $30^{\circ} \mathrm{C}$ and $37^{\circ} \mathrm{C}$ and daily observed for three weeks. The yeast like colonies obtained after 48 hours, were identified thanks to the germination serum test; to the test of chlamydosporulation on PCB medium (Bio-Rad) and to the auxanogram with the Auxacolor kit Tm2 (Bio-Rad). The filamentous colonies were identified on the basis of macroscopic and microscopic features after coloring with blue cotton.

\section{Data Typing and Analysis}

The data have been typed on Excel and analyzed thanks to the software Epi Info 6.04fr (CDC Atlanta, United States) The information about age, sex, sample date, clinical signs and diagnosis mentioned and the mycological screening result were gathered for each patient. The data were described in terms of average or percentage whether it was about quantitative or qualitative variables. The prevalence rates were calculated as a whole but also according to age, sex, month and the reason of medical checking. The statistical comparisons were made using the $\mathrm{CHI}-2$ test, or the Fisher test according to the feasibility conditions with a significant $5 \%$ limit.

\section{Ethical Considerations}

Confidentiality has been respected in the data gathering and analysis by giving to each patient an anonymous number.

\section{Results}

During the period of the survey, 136 patients checked at the Otorhinolaryngology clinic of Fann hospital benefited from a mycological check because they were suspected to suffer from otomycosis. Their ages vary from 2 months to 86 years with an average age of 32.8 years. The adult patients aged above 15 years represented $82.1 \%$ of the target population while the children under 15 years were 24 , that is to say $17.9 \%$ of the total number. The sex ratio $\mathrm{M} / \mathrm{F}$ of the patients checked was about 0.74 in favor of female gender with $57.4 \%$ of female gender patients against $42.6 \%$ of male gender. We noticed a slight increase of patients during the month of May. The rainy season going from July to October, the other months representing the dry season, 111 patients were reordered during the dry season against 25 during the rainy season.

On the clinical plan, symptomatology was indicated for 111 patients. It was about pruritus (45.9\%), otorrhea (26.1\%), otalgia (17.1\%), and hypoacusis $(11.7 \%)$. For 16 patients the request of mycological check was justified by a diagnosis of earache among which 6 were chronic and 4 were malignant. The diagnosis of otomycosis was clinically proved among 4 patients and for 5 patients the reason of the request was not mentioned. Out of the 136 samples checked at the lab, 59 were positive to the direct screening and/or culture, that is to say an overall prevalence of $43.4 \%$. We noticed two negative results to the direct screening with a positive culture. Inversely, the direct checking was positive by visualizing aspergillary heads and thin septate hyphae ramified with an acute angle but with a negative culture among two of the cases. The direct microscopic examination and the culture were positive simultaneously for 55 auricular samples that is say $40.4 \%$.

The number of positive cases described in table 1 , was more important among patients aged from 20 to 39 $(35.1 \%)$ and the patients over 60 years old $(24.6 \%)$. The differentiation of the prevalence rates according to age has proved that the percentages varied from $33.33 \%$ among the patients aged between 5 and 9 to $63.6 \%$ among patients over 60 years of age (Table 1). However, the variations noticed were not statistically meaningful $(p=0,204)$. As far as gender 
is concerned, we have noticed that the prevalence rate of otomycosis was significantly higher female patients $(52.6 \%)$ than among male subjects (31\%) representing $p=0,01$ (Table 1). Depending on the months, the prevalence rates did not vary significantly (Table 1) but there was a statically significant difference of the noticed rates between January
(25\%) and July and August combined (80\%) $(\mathrm{p}=0,009)$. The percentage of positive cases was higher during the rainy season which goes in Dakar from July to October (56\%) than during the dry season (40.5\%) but that difference was not significative $(p=0,15)$.

\begin{tabular}{|c|c|c|c|}
\hline Age Range (Year)* & $\begin{array}{l}\text { Number of Patients } \\
\text { Examinated (N) }\end{array}$ & Number of Positive Cases n (\%) & Prevalence Rate $\% \mathrm{n} / \mathrm{N}$ \\
\hline$<2$ & 7 & $4(7)$ & 57.1 \\
\hline $2-4$ & 7 & $4(7)$ & 57.1 \\
\hline $5-9$ & 6 & $2(3.5)$ & 33.3 \\
\hline $10-14$ & 4 & $0(0)$ & 0 \\
\hline $15-19$ & 10 & $3(5.3)$ & 30 \\
\hline $20-39$ & 55 & $20(35.1)$ & 36.4 \\
\hline $40-59$ & 23 & $10(17.5)$ & 43.5 \\
\hline$>60$ & 22 & $14(24.6)$ & 63.6 \\
\hline Total & 134 & $57(100)$ & 42.5 \\
\hline \multicolumn{4}{|c|}{ Sexe** } \\
\hline Male & 58 & $18(30.5)$ & 31 \\
\hline Female & 78 & $41(69.5)$ & 52.6 \\
\hline Total & 136 & $59(100)$ & 43.4 \\
\hline \multicolumn{4}{|c|}{ Period of Sampling (Month) $* * *$} \\
\hline October & 12 & $5(8,5)$ & 41,7 \\
\hline November & 12 & $6(10,2)$ & 50 \\
\hline December & 16 & $5(8.5)$ & 31.2 \\
\hline January & 16 & $4(6.8)$ & 25 \\
\hline February & 9 & $4(6.8)$ & 44.4 \\
\hline March & 16 & $6(10.2)$ & 37.5 \\
\hline April & 17 & $9(15.2)$ & 52.9 \\
\hline May & 19 & $7(11.8)$ & 36.8 \\
\hline June & 6 & $4(6.8)$ & 66.7 \\
\hline July & 1 & $1(1.7)$ & 100 \\
\hline August & 9 & $7(11.8)$ & 77.8 \\
\hline September & 3 & $1(1.7)$ & 33.3 \\
\hline Total & 136 & $59(100)$ & 43.4 \\
\hline
\end{tabular}

$* \mathrm{p}=0.204$ Fisher test

** $\mathrm{p}=0.0122 ; \mathrm{ddl}=1$

$* * * \mathrm{p}=0.411$ Fisher test

Table 1: Otomycosis prevalence rates according to the socio-demographic characteristics.

On the clinical plan, the prevalence rate was significantly higher $(72.2 \%)$ among patients suffering from otalgia (Table 2). 


\section{Open Access Journal of Mycology \& Mycological Sciences}

\begin{tabular}{|c|c|c|c|}
\hline Indication & $\begin{array}{c}\text { Number of Patients } \\
\text { Examinated (N) }\end{array}$ & $\begin{array}{c}\text { Number of Positive Cases } \\
\mathbf{n ~ ( \% )}\end{array}$ & Prevalence Rate \% n/N \\
\hline Auricular pruritus & 48 & $18(30.5)$ & 37.5 \\
\hline Hypo-acousis & 13 & $4(6.8)$ & 30.8 \\
\hline Otorrhea & 29 & $10(16.9)$ & 34.5 \\
\hline Otalgia & 18 & $13(22)$ & 72.2 \\
\hline Otagia and Auricularl pruritus & 1 & $1(1.7)$ & 100 \\
\hline Chronic otitis & 6 & $1(1.7)$ & 16.7 \\
\hline Malignant otitis & 4 & $3(5.1)$ & 50 \\
\hline Otitis & 6 & $3(5,1)$ & 50 \\
\hline Itchy otitis & 2 & $1(1.7)$ & 75 \\
\hline Otomycosis & 4 & $3(5.1)$ & 40 \\
\hline Non notified & 5 & $2(3.4)$ & 43.4 \\
\hline Total & 136 & $59(100)$ & 5 \\
\hline
\end{tabular}

Table 2: Prevalence rate of otomycoses according to the indication of the prescription of the mycological examination. $\mathrm{p}=0,063$ Test de Fisher

Concerning the 59 positive samples, species of the genus Aspergillus were the etiological agents of $55.9 \%$ of cases and those like Candida in $44.1 \%$ of the cases. Among the 57 positive cultures, Aspergillus fumigatus and Candida albicans were the most recurrent species representing each of them $26.3 \%$ of the fungal spectrum alone followed by Aspergillus niger $(22.8 \%)$. The other isolated species were weakly represented (Figure 1).

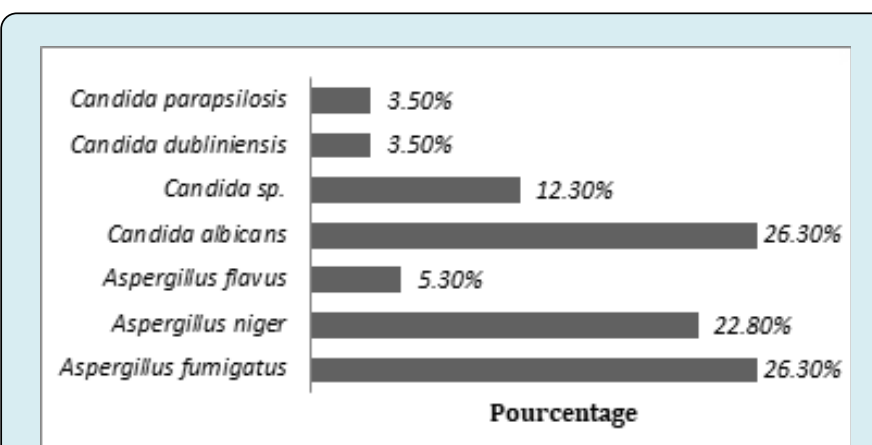

Figure 1: Distribution of etiological agents isolated in culture from patients with otomycosis.

There was no association of fungal species. We have noticed two cases of direct microscopic examination objectifying the Aspergillary heads without being able to make a precise identification of the Aspergillus species because the culture was revealed to be negative.

\section{Discussion}

The mycological diagnosis has a paramount importance in the cure of otomycosis because it permits to channel the choice of the treatment. The survey has enabled us to determine the prevalence (43.4\%) of this disease usually put aside in the routine daily practice. The mycological diagnosis has then rebutted an otomycosis among (56.6\%) of patients suspected to suffer clinically from fungal earache and therefore to avoid them from being given a treatment against fungi. This shows the interest of the mycological diagnosis to establish a sure diagnosis of this mycosis.

The prevalence rate we obtained is comparable to that registered by other authors in Africa among patients suffering from earaches. It is the cases for figures reported in Abidjan in Ivory Coast with prevalence rates ranging from $4.6 \%$ [8] $46.5 \%$ [9] to $80 \%$ depending on the years and the survey setting. In Morocco, prevalence rates of $41 \%$ have been reported in Rabat [1] and 36.5\% in Marrakech [10]. These data show that fungal infections have an important place in the earache etiologies in Africa. On the contrary, prevalence rates which are higher or lower have been reported out of the continent: $30.4 \%$ in Poland [11], 19.4\% in Brazil [12], $28.4 \%$ in Spain [7] and 69\% in Iran [13]. The high prevalence noticed in Senegal and in other African countries like Ivory Coast [14] can be explained by the combination of some factors: weather (moisture and heat), some religious, cultural or aesthetical practices causing to keep the external ear canal wet (ablutions, wearing scarf or veil, long braided wick made of synthetical or real hair by women). The excessive cleaning of ears using cotton buds or unadapted tools such as sticks, hairpins or poultry feathers also cause the emergence of this fungal otitis. The immunodepression caused by HIV/ AIDS, corticotherapy and antibiotics therapy has also been held responsible for the earaches with fungi [15]. In this study, apart from one case of HIV positive that has developed a Candida albicans otomycosis; the HIV immunity status of the patients was not mentioned. However we registered two positive cases, the one with Candida albicans and the other 


\section{Open Access Journal of Mycology \& Mycological Sciences}

with Candida $s p$ among two patients suffering from diabetes presenting a malignant otitis. Diabetes predisposing to the development of candidiasis because of the lowering of immunity might have been a factor favoring the fungal infection.

In the distribution of cases according to age, the prevalence rates do not vary significantly. However among patients aged 15 and more, we have noticed a steady increase of the prevalence with age with a large predominance among patients aged over 60 . The otomycosis seem therefore to be more an infection of the adult patient as it is shown by the results obtained in Morocco [1] and in India [2]. As far as gender is concerned, we have registered more cases among women than men with a statically significant difference. Similar results have been reported also in Shanghai China [17] with a gender ratio F/M of 2.1, in Morocco in Rabat [1] with 23 cases of otomycosis among women against 6 among men and in Marrakech [10] with a gender ratio of 1.2, but also in Nigeria with $61.64 \%$ of women against $38.36 \%$ of men [18]. This predominance of otomycosis among women in Senegal and in these countries might be explained by the dressing habits in relation to fashion or the social or even religious customs. Actually, it is common for women to cover their heads with a head tie commonly called « head handkerchiefs ». Some wear veils and others wear long synthetic wigs or real hair ones. These hairstyles by keeping the ears tight favor the moisturing of the hair which becomes more conducive to the development of mycosis especially during the hot and damp season of the year. These weather conditions could explain the highest prevalence rates noticed during the rainy season when the conditions of heat and moisture are gathered.

On the clinical plan, the positivity rate of the mycological examination was significantly higher among patients suffering from earache.. However the auricular pruritus was the predominant clinical sign among the diagnosed patients. So, the biological diagnosis is important to confirm any clinical suspicion of fungal mycosis, the noticed clinical signs being not pathognomonic. In fact, it has been described that otalgia was one of the most recurrent symptoms in the case of serious form of otomycosis [19]. The other signs of the clinical table are closer to those of the usual external earache, with a less important intensity.

Among the isolated pathogenic agents, the filamentous fungi, namely the moulds were more represented than the yeasts. Aspergillus fumigatus has been the most recurrent species followed with Candida albicans and Aspergillus niger in our series of cases. However, some Candida strains were not identified at the stage of species because we were facing a shortage of Auxacolor reactives kit TM2 (Bio-Rad).
As for the Aspergillus genus, the observation of the Aspergillary head from the direct microscopic examination of the auricular sample without any positive culture among two patients does not allow to identify the species. The negative culture could be explained by the fact that the seeds were not enough or were even dead. The aspergillary heads can be observed in the superficial air sites such as the sinus or the external hearing canal. The question is to know if it is colonization or an infection. In both cases of our study, the direct microscopic screening, apart from the aspergillay heads, have revealed the presence of septate hyphae with an acute angle of the aspergillary type and the clinical indication of the demand of a mycological checking was for one of the patients a suspicion of aspergillosis and for the other one, it was a purulent otorrhea with greenish filamentous depots after an otological test. These clinical data associated to the mycological results are in favour of a real otomycosis due to Aspergillus even if the culture has proven to be negative.

This distribution of species varies however, from one country to another, one region to another. Actually, the reported figures from other regions of tropics and subtropical zones reveal, for example in Morocco that Aspergillus niger has been the dominant species in two different surveys with a $35 \%$ prevalence $[1,10]$. Similar results have been noticed in Nigeria [18] and in China [17] with A. niger representing respectively $48.35 \%$ and $54.78 \%$ of the agents responsible for fungal otiti. In Ivory Coast, it was Aspergillus followed by Candida guilliermondi [14], whereas the Candida genus dominated in Poland [11] and in Brazil [12]. In all cases, the predominance of species of the Aspergillus and Candida genus make us favour the imidazole derivatives, to a large spectrum, the econazole type, ketoconazole or itraconazole in the therapeutical treatment as it was suggested by other authors $[19,20,21]$.

\section{Conclusion}

This survey has enabled to show the relatively important prevalence of otomycosis cases in the hospital environment in Senegal. The predominance of Aspergillus and Candida genus in the etiological agents of average earaches make it necessary to have recourse to a mycological diagnosis in order to avoid the excessive use of antibiotics. It would be important to make, with the view to having prevalence surveys on wider samples in order to better assess the impact of these fungal pathologies and the risks they represent.

\section{References}

1. Aboulmakarim S, Tligui H, El Mrini M, Zakaria I, Handour $\mathrm{N}$, et al. (2010) Otomycosis: Clinical and Mycological study of 70 cases. J Mycol Med 20: 48-52.

2. Aneja KR, Sharma C, Joshi R (2010) Fungal infection of 


\section{Open Access Journal of Mycology \& Mycological Sciences}

the ear: a common problem in the north eastern part of Haryana. Int J Pediatr Otorhinolaryngol 74(6): 604-607.

3. Wang MC, Liu CY, Shiao AS, Wang T (2005) Ear problems in swimmers. J Chin Med Assoc 68(8): 347-352.

4. Viswanatha B, Naseeruddin K (2011) Fungal infections of the ear in immunocompromised host: a review. Mediterr J Hematol Infect Dis 3(1): e2011003.

5. Vennewald I, Klemm E (2010) Otomycosis: Diagnosis and treatment. Clin Dermatol 28(2): 202-211.

6. Dubach P, Mantokoudis G, Caversaccio M (2010) Ear canal cholesteatoma: meta-analysis of clinical characteristics with update on classification, staging and treatment. Curr Opin Otolaryngol Head Neck Surg 18(5): 369-376.

7. García-Agudo L, Aznar-Marín P, Galán-Sánchez F, GarcíaMartos P, Marín-Casanova P, et al. (2011) Otomycosis due to filamentous fungi. Mycopathologia 172(4): 307-310.

8. Yavo W, Kassi RR, Kiki-Barro PC, Bamba A, Kplé T, et al. (2004) Prevalence and risk factors for otomycosis treated in the hospital setting in Abidjan (Ivory Coast). Med Trop 64(1): 39-42.

9. Djohan V, Vanga-Bosson HA, Yavo W, Kiki-Barro PMC, Konate A, et al. (2010) Prévalence et facteurs de risque des otomycoses à Abidjan (Côte d'Ivoire). Eur J Sci Res 40: $232-238$.

10. El Mezouari EM, Mouhib H, Darouassi Y, Ammar H, Moutaj R (2017) Otomycosis in Marrakech: Study of 19 cases. International Journal of Infectious Diseases and therapy 2(1): 1-3.

11. Kurnatowski P, Filipiak A (2001) Otomycosis: prevalence, clinical symptoms, therapeutic procedure Mycoses
44(11-12): 472-479.

12. Pontes ZB, Silva AD, Ede OL, Mde HG, Oliveira NM, et al. (2009) Otomycosis: a retrospective study. Braz J Otorhinolaryngol 75(3): 367-370.

13. Barati B, Okhovvat SA, Goljanian A, Omrani MR (2011) Otomycosis in central Iran: a clinical and mycological study. Iran Red Crescent Med J 13(12): 873-861.

14. Adoubryn KD, N'Gattia VK, Kouadio-Yapo GC, Nigué L, Zika DK, et al. (2014) Epidemiology of otomycoses at the University Hospital of Yopougon (Abidjan-Ivory Coast). J Mycol Med 24(2): 9-15.

15. Kazemi A, Majidinia M, Jaafari A, Mousavi ASA, Mahmoudabadi ZA, et al. (2015) Etiologic Agents of Otomycosis in the North-Western Area of Iran. Jundishapur J Microbiol 8(9): e21776.

16. Jia X, Liang Q Chi F, Cao W (2012) Otomycosis in Shanghai: aetiology, clinical features and therapy. Mycoses 55(5): 404-409.

17. Fasunla J, Ibekwe T, Onakoya $P$ (2008) Otomycosis in western Nigeria. Mycoses 51(1): 67-70.

18. Bourée P, Dahane N (2011) Otites: penser aux champignons. Option Bio 22(462): 22-23.

19. Kaya AD, Kiraz $N$ (2007) In vitro susceptibilities of Aspergillus spp. causing otomycosis to amphotericin B, voriconazole and itraconazole. Mycoses 50(6): 447-450.

20. Szigeti G, Sedaghati E, Mahmoudabadi AZ, Naseri A, Kocsubé S, et al. (2011) Species assignment and antifungal susceptibilities of black aspergilli recovered from otomycosis cases in Iran. Mycoses 55(4): 333-338.

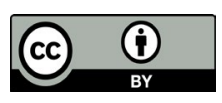

